\title{
Bottom friction effects on linear wave propagation
}

\author{
G. Simarro ${ }^{\mathrm{a}, *}$, A. Orfila ${ }^{\mathrm{b}}$, A. Galán ${ }^{\mathrm{a}, \mathrm{b}}$, G. Zarruk ${ }^{\mathrm{b}}$. \\ ${ }^{a}$ E.T.S.I. Caminos, Canales y Puertos, Universidad de Castilla-La Mancha. \\ 13071 Ciudad Real, Spain. \\ b IMEDEA (CSIC-UIB). 07190 Esporles, Spain
}

\begin{abstract}
Bottom boundary layer effects on the linear wave propagation over mild slope bottoms are analyzed. A modified WKB approximation is presented including boundary layer effects. Within the boundary layer, two cases are considered: laminar (constant viscosity) and turbulent. Boundary layer effects are introduced by coupling the velocity inside the boundary layer to the irrotational velocity in the core region through the bottom boundary condition. This formulation properly accounts for the phase between near bed velocity and bed shear stress. The resulting differential equation for the energy conservation introduces a new term accounting for the energy losses due to the boundary layer effects.
\end{abstract}

Key words: Linear theory, Boundary layer, WKB Approximation.

\section{Introduction}

The bottom boundary layer in water wave propagation is important, at least, in two fundamental aspects related to coastal and environmental engineering. First, it determines the stress that the water transmits to the bottom, which is important in the near shore morphodynamics and ecosystems, since bottom shear stress is responsible for sediment transport (Fredsoe and Deigaard, 1992; Nielsen, 1992).

Secondly, the energy dissipation in the boundary layer is responsible for wave damping, modifying not only the wave amplitude but also wave celerity and

* Corresponding author. Phone(+34) 926295300

Email address: gonzalo.simarro@uclm.es (G. Simarro). 
phase. Boundary layer effects can be of first order importance if propagation over long distances is considered. Therefore, in order to obtain accurate models for water waves propagation, the energy dissipation within the boundary layer has to be taken into account in their formulation. This is often done by introducing the bed shear stress in the horizontal momentum equation. The shear stress transmitted to the bottom is usually expressed as the square of the near bottom velocity as (Dingemans, 1997; Svendsen, 2005),

$$
\boldsymbol{\tau}=\rho C_{f} \mathbf{u}|\mathbf{u}|
$$

where $\boldsymbol{\tau}$ is the bottom shear stress, $\rho$ is the water density, $\mathbf{u}$ is the near bed velocity and $C_{f}$ is a dimensionless friction coefficient, which is a function of a relative bed roughness and a Reynolds number (Fredsoe and Deigaard, 1992). As long as $C_{f}$ is properly chosen, the above frictional model is appropriate if the primary concern is the amount of energy dissipated in a time scale bigger than one wave period. However, this bottom stress model does not correctly describe the phase of the bottom stress relative to the bottom velocity, since it is well known, for instance, that the bottom stress is $\pi / 4$ out of phase with the bottom velocity for an oscillatory laminar boundary layer (Nielsen, 1992). This phase lag is smaller in the turbulent case (Fredsoe and Deigaard, 1992). A bottom stress such as the above described is not adequate when computing sediment transport rate, unless an empirical phase shift is introduced.

For the laminar boundary layer case and linear water wave propagation, some results introducing the proper phase have already been obtained (Booij, 1981; Dalrymple et al., 1984). More recently Liu and Orfila (2004) introduced the result of integrating the linearized and laminar boundary layer into the bottom boundary condition for the potential in the core, obtaining a set of Boussinesqtype equations including the bottom laminar boundary layer effects. The boundary layer effects were introduced in the continuity equation as a convolution integral. The extension for uneven bottoms was derived by Liu et al. (2006) for mild slope conditions. The above derivations were made under the assumption of a laminar or constant viscosity.

Sometimes the model of constant eddy viscosity is used to describe the bottom boundary layer and, further, the eddy viscosity is modeled as a linear function of the distance to the bottom (Kajiura, 1964; Grant and Madsen, 1979; Simarro et al., 2008). Following this concept, Orfila et al. (2007) extended the formulation by Liu et al. (2006) to include the effects of a fully developed turbulent bottom boundary layer in a nonlinear wave propagation model. In this work, we estimate the effects of the bottom friction within the framework of such a model leaving aside the accuracy of its performance. In reality the turbulent boundary layer is certainly nonlinear.

In many real situations, water wave linear theory is accurate enough for propagating waves from deep to shallow water, so that simulations can be done 
in a much more efficient way. The aim of this paper is to introduce the above mentioned boundary layer formulations (laminar and turbulent) in linear models. Since the goal is to present the influence of the boundary layer, numerical results will only be presented in one dimensional cases.

The paper is structured in the following manner. For completeness, we summarize the governing equations, boundary conditions and the Fourier expansion for periodic waves in Section 2. The equations for the WKB approximation are derived in Section 3. The leading order solution provides the modified equation for the energy conservation where the dissipation effects are included. These set of equations are expressed in terms of the Fourier (harmonic) components of the free surface amplitude. A brief discussion of the results is presented in Section 4.

\section{Governing equations}

Hereinafter, dimensional variables are primed and dimensionless variables are unprimed. We will consider the domain between the sea bed, which is located at $z^{\prime}=-h^{\prime}\left(x^{\prime}, y^{\prime}\right)$, and the free surface at $z^{\prime}=\eta^{\prime}\left(x^{\prime}, y^{\prime}\right)$ (Figure 1). The Mean Water Level (MWL) is considered to be at $z^{\prime}=0$.

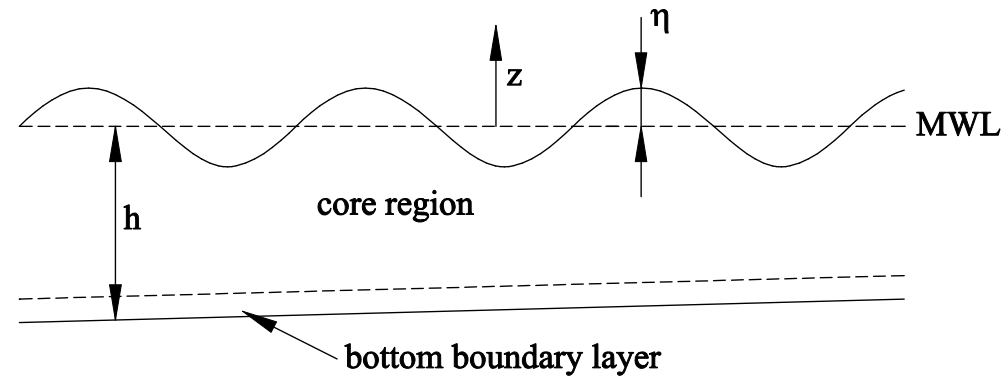

Fig. 1. Illustration of the variables and the boundary conditions for the 2 dimensional wave propagation problem.

For convenience, here $\boldsymbol{\nabla}^{\prime}$ will stand for $\left(\partial / \partial x^{\prime}, \partial / \partial y^{\prime}\right)$ and $\mathbf{a}^{\prime}$ for $\left(a_{x}^{\prime}, a_{y}^{\prime}\right)$. The no flux boundary conditions at the bottom and the free surface are obtained considering that they are material surfaces. These conditions read

$$
\begin{array}{ll}
\frac{\partial h^{\prime}}{\partial t^{\prime}}+\mathbf{u}^{\prime} \cdot \nabla^{\prime} h^{\prime}+w^{\prime}=0, & z^{\prime}=-h^{\prime} \\
\frac{\partial \eta^{\prime}}{\partial t^{\prime}}+\mathbf{u}^{\prime} \cdot \nabla^{\prime} \eta^{\prime}-w^{\prime}=0, & z^{\prime}=\eta^{\prime}
\end{array}
$$

where $\mathbf{u}^{\prime}$ is the horizontal velocity, $w^{\prime}$ is the vertical component and $t^{\prime}$ is time.

In water wave propagation problems, it is usually assumed that the fluid is inviscid, so that it is allowed to slip over the contours. In that case, the no 
flux conditions in expressions (1) are the only kinematic conditions to be used. Moreover, the velocity is considered to be irrotational, i.e., there exists a velocity potential $\Phi^{\prime}$ so that

$$
\mathbf{u}^{\prime}=\nabla^{\prime} \Phi^{\prime}, \quad \text { and } \quad w^{\prime}=\frac{\partial \Phi^{\prime}}{\partial z^{\prime}}
$$

For incompressible fluids, the continuity equation for the potential reads

$$
\nabla^{\prime 2} \Phi^{\prime}+\frac{\partial^{2} \Phi^{\prime}}{\partial z^{\prime 2}}=0, \quad-h^{\prime} \leqslant z^{\prime} \leqslant \eta^{\prime}
$$

and the boundary conditions (1) for rigid bed (i.e., $\left.\partial h^{\prime} / \partial t^{\prime}=0\right)$ are,

$$
\begin{aligned}
\nabla^{\prime} \Phi^{\prime} \cdot \nabla^{\prime} h^{\prime}+\frac{\partial \Phi^{\prime}}{\partial z^{\prime}} & =0, & z^{\prime} & =-h^{\prime} \\
\frac{\partial \eta^{\prime}}{\partial t^{\prime}}+\nabla^{\prime} \Phi^{\prime} \cdot \nabla^{\prime} \eta^{\prime}-\frac{\partial \Phi^{\prime}}{\partial z^{\prime}} & =0, & z^{\prime} & =\eta^{\prime},
\end{aligned}
$$

The dynamic free surface boundary condition can be obtained from Bernoulli's equation imposing the continuity of the pressure field at the water-air interface. Assuming constant atmospheric pressure, it reads

$$
\frac{\partial \Phi^{\prime}}{\partial t^{\prime}}+\frac{1}{2}\left\{\nabla^{\prime} \Phi^{\prime} \cdot \nabla^{\prime} \Phi^{\prime}+\frac{\partial \Phi^{\prime}}{\partial z^{\prime}} \frac{\partial \Phi^{\prime}}{\partial z^{\prime}}\right\}+g^{\prime} \eta^{\prime}=0, \quad z^{\prime}=\eta^{\prime},
$$

being $g^{\prime}$ the acceleration of gravity.

\subsection{Dimensionless equations}

The above equations are scaled by considering usual dimensionless variables

$$
\{z, h\} \equiv \frac{1}{h_{0}^{\prime}}\left\{z^{\prime}, h^{\prime}\right\}, \quad \eta \equiv \frac{\eta^{\prime}}{a_{0}^{\prime}}, \quad\{x, y\} \equiv k_{0}^{\prime}\left\{x^{\prime}, y^{\prime}\right\}
$$

and

$$
\Phi \equiv \frac{k_{0}^{\prime} h_{0}^{\prime}}{a_{0}^{\prime} \sqrt{g^{\prime} h_{0}^{\prime}}} \Phi^{\prime}, \quad \mathbf{u} \equiv \frac{h_{0}^{\prime}}{a_{0}^{\prime} \sqrt{g^{\prime} h_{0}^{\prime}}} \mathbf{u}^{\prime}, \quad w \equiv \frac{k_{0}^{\prime} h_{0}^{\prime 2}}{a_{0}^{\prime} \sqrt{g^{\prime} h_{0}^{\prime}}} w^{\prime}, \quad t \equiv k_{0}^{\prime} \sqrt{g^{\prime} h_{0}^{\prime}} t^{\prime},
$$

with $a_{0}^{\prime}$ and $h_{0}^{\prime}$ being characteristic lengths for the wave amplitude and the water depth respectively and $k_{0}^{\prime}$ is the characteristic wave number. The boundary 
value problem defined in equations (3) reads, in dimensionless form

$$
\begin{array}{rlrl}
\nabla^{2} \Phi+\frac{1}{\mu^{2}} \frac{\partial^{2} \Phi}{\partial z^{2}} & =0, & -h \leqslant z \leqslant \epsilon \eta, \\
\nabla \Phi \cdot \nabla h+\frac{1}{\mu^{2}} \frac{\partial \Phi}{\partial z} & =0, & z=-h, \\
\frac{\partial \eta}{\partial t}+\epsilon \nabla \Phi \cdot \nabla \eta-\frac{1}{\mu^{2}} \frac{\partial \Phi}{\partial z} & =0, & z=\epsilon \eta, \\
\frac{\partial \Phi}{\partial t}+\frac{\epsilon}{2}\left\{\nabla \Phi \cdot \nabla \Phi+\frac{1}{\mu^{2}} \frac{\partial \Phi}{\partial z} \frac{\partial \Phi}{\partial z}\right\}+\eta & =0, & z & =\epsilon \eta,
\end{array}
$$

where

$$
\epsilon \equiv \frac{a_{0}^{\prime}}{h_{0}^{\prime}}, \quad \text { and } \quad \mu \equiv k_{0}^{\prime} h_{0}^{\prime}
$$

are the dimensionless parameters representing, respectively, the nonlinear and the dispersive effects. Throughout this paper only linear waves are to be analyzed and, hence, hereafter it will be considered that $\epsilon \equiv 0$. The dispersive parameter, $\mu$, is small in shallow water conditions, but throughout this work no assumption about its value will be made.

\subsection{The boundary layer}

In real fluids, viscous effects become important within a thin layer attached to the bottom (Figure 1), where velocity gradients are large. Therefore, the hypothesis of inviscid fluid is no longer valid in this region (boundary layer) and rotational and irrotational velocity components have to be considered, i.e.

$$
\mathbf{u}=\nabla \Phi+\mathbf{u}_{\mathbf{r}}, \quad \text { and } \quad w=\frac{\partial \Phi}{\partial z}+w_{r}
$$

with $\mathbf{u}_{\mathbf{r}}$ and $w_{r}$ standing, respectively, for the horizontal and vertical components of the rotational velocity. The no flux boundary condition at the bottom in (4b) reads now

$$
\left(\boldsymbol{\nabla} \Phi+\mathbf{u}_{\mathbf{r}}\right) \cdot \boldsymbol{\nabla} h+\frac{1}{\mu^{2}} \frac{\partial \Phi}{\partial z}+\frac{w_{r}}{\mu^{2}}=0, \quad z=-h
$$

Following Liu et al. (2006), to solve the rotational velocity at the seabed we will consider a coordinate system locally parallel to the bed (hats in Figure 2). We note that the rotational velocity component normal to the bottom $\left(\hat{w}_{r}\right)$ is

$$
\hat{w}_{r}=w_{r}\left(1+\mathcal{O}\left((\mu \nabla h)^{2}\right)\right)+\mu^{2} \mathbf{u}_{\mathbf{r}} \cdot \nabla h
$$




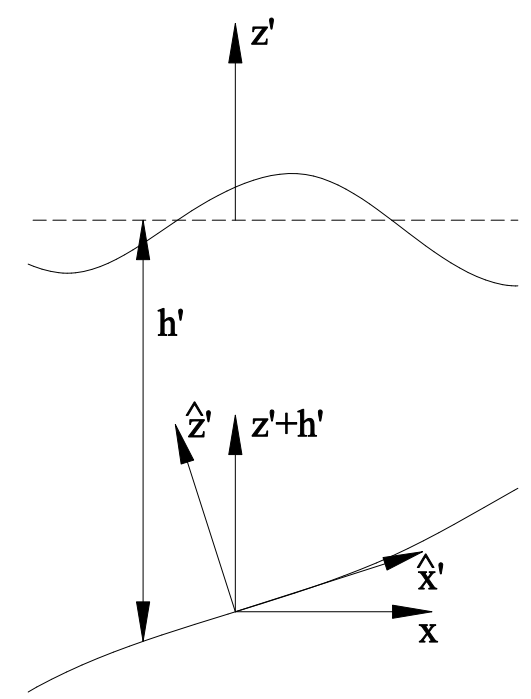

Fig. 2. Schematic view of the coordinate system locally parallel to the bed.

where $\mathcal{O}(\nabla h) \ll \mathcal{O}(1)$ since mild slope conditions will be assumed. Therefore, the expression (6) is also

$$
\nabla \Phi \cdot \nabla h+\frac{1}{\mu^{2}} \frac{\partial \Phi}{\partial z}+\frac{\hat{w}_{r}}{\mu^{2}}=0, \quad z=-h .
$$

Above expression (7) introduces the term $\hat{w}_{r} / \mu^{2}$ which accounts for the boundary layer effects (compare to expression (4b)).

The result for $\hat{w}_{r}$ at $z=-h$ is, for the laminar case (Liu and Orfila, 2004)

$$
\hat{w}_{r}(z=-h)=-\frac{\chi \mu^{2}}{\sqrt{\pi}} \int_{0}^{t} \frac{\nabla^{2} \Phi(z=-h, t=\xi)}{\sqrt{t-\xi}} d \xi,
$$

where $\chi$ is a dimensionless parameter accounting for the boundary layer strength and defined as

$$
\chi \equiv \frac{1}{h_{0}^{\prime}} \sqrt{\frac{\nu^{\prime}}{k_{0}^{\prime} \sqrt{g^{\prime} h_{0}^{\prime}}}} \ll 1,
$$

with $\nu^{\prime}$ being the kinematic viscosity, which is constant for the laminar case.

\section{Turbulent boundary layer for periodic waves}

As mentioned, boundary layer is usually turbulent in real cases. In the case of turbulent boundary layer, the problem is much more complex. The constant eddy viscosity linear model (Kajiura, 1964; Grant and Madsen, 1979) has been used often to describe the bottom boundary layer. In this paper we use such model leaving aside the boundary layer nonlinearity issue. 
Considering the flow periodic in time, any time dependent function $\xi$ can be expressed as

$$
\xi=\frac{1}{2} \sum_{n} \xi_{n} \exp (-i n \omega t)=\frac{\xi_{0}}{2}+\sum_{n>1} \Re\left\{\xi_{n} \exp (-i n \omega t)\right\}
$$

with $\omega$ being the main frequency and $\xi_{n}$ the Fourier components. The solution for $\hat{w}_{r}$ can be written in compact form for both the laminar and the turbulent cases as (Simarro and Orfila, 2007)

$$
\hat{w}_{r, n}(z=-h)=-\chi \mu^{2} \nabla^{2} \Phi_{n}(z=-h) \frac{1+i}{\sqrt{2 n \omega}} \vartheta_{n}
$$

where

$$
\vartheta_{n}= \begin{cases}1, & \text { laminar BL; } \\ \sqrt{\hat{z}_{0}} \frac{K_{1}\left(2 \sqrt{-i n \omega \hat{z}_{0}}\right)}{K_{0}\left(2 \sqrt{-i n \omega \hat{z}_{0}}\right)}, & \text { turbulent BL }\end{cases}
$$

and with $\hat{z}_{0}$ defined as

$$
\hat{z}_{0} \equiv \frac{1}{\chi} \frac{\hat{z}_{0}^{\prime}}{h_{0}^{\prime}}
$$

where $\hat{z}_{0}^{\prime}$ is the elevation over the seabed where the velocity cancels.

The elevation $\hat{z}_{0}$ depends on the bed roughness and a Reynolds number. Besides, the parameter $\chi$ is given in equation (8): in the case of turbulent boundary layer (which is the usual one), $\nu^{\prime}$ must be replaced by a characteristic kinematic viscosity, which depends on the own solution. The solution is, therefore, to be obtained in a iterative way (Orfila et al., 2007). The performance of this boundary layer model in terms of the friction factor can be found in Simarro et al. (2008).

\subsection{Potential equations for periodic waves}

Since the above boundary layer results were presented in terms of the Fourier components, the equations for the core region will be hereinafter presented in terms of Fourier components (assuming that the movement is periodic).

Recalling expression (9), the above equations (4) read now, replacing (4b) by 
(7) and linearizing (i.e., setting $\epsilon \equiv 0$ )

$$
\begin{aligned}
\nabla^{2} \Phi_{n}+\frac{1}{\mu^{2}} \frac{\partial^{2} \Phi_{n}}{\partial z^{2}} & =0, & & -h \leqslant z \leqslant 0, \\
\nabla \Phi_{n} \cdot \nabla h+\frac{1}{\mu^{2}} \frac{\partial \Phi_{n}}{\partial z}-\chi \nabla^{2} \Phi_{n} \frac{1+i}{\sqrt{2 n \omega}} \vartheta_{n} & =0, & & z=-h, \\
-i n \omega \eta_{n}-\frac{1}{\mu^{2}} \frac{\partial \Phi_{n}}{\partial z} & =0, & & z=0, \\
-i n \omega \Phi_{n}+\eta_{n} & =0, & & z=0 .
\end{aligned}
$$

We remark that the boundary layer effects appear only at the bottom boundary condition (12b). Besides, combining the boundary conditions at the free surface we get

$$
\frac{1}{\mu^{2}} \frac{\partial \Phi_{n}}{\partial z}-n^{2} \omega^{2} \Phi_{n}=0, \quad z=0
$$

Since the problem is linear, there is no interaction between different harmonics and, hereafter, only $n=1$ will be considered (and $n$ will be omitted). Further, in order to solve the potential, only equations (12a), (12b) and (13) are to be used, i.e.

$$
\begin{aligned}
\nabla^{2} \Phi+\frac{1}{\mu^{2}} \frac{\partial^{2} \Phi}{\partial z^{2}} & =0, & -h & \leqslant z \leqslant 0, \\
\nabla \Phi \cdot \nabla h+\frac{1}{\mu^{2}} \frac{\partial \Phi}{\partial z}-\chi \Omega \nabla^{2} \Phi & =0, & z & =-h, \\
\frac{1}{\mu^{2}} \frac{\partial \Phi}{\partial z}-\omega^{2} \Phi & =0, & z & =0,
\end{aligned}
$$

with

$$
\Omega \equiv \frac{1+i}{\sqrt{2 \omega}} \vartheta
$$

Once the potential is solved, the free surface elevation can be computed from equation (12d), i.e.

$$
\eta=i \omega \Phi, \quad z=0
$$

The above equations (14) are solved in the following section using the WKB approximation.

\section{The WKB approximation}

The WKB is usually employed in water wave propagation to handle with the fact that there are two characteristic length scales: one corresponding to the wave length and another corresponding to the bottom variations. 
Here, three different horizontal characteristic lengths will be present: the two above mentioned plus the one corresponding to the boundary layer effects on the wave damping.

For the sake of clarity, we consider first the case when the bed is flat. As above mentioned, in this case there are two different scales (the wave length, which is order 1 according to the scaling, and the characteristic damping scale, which will be order $\chi^{-1}$ ). The WKB approximation can be here performed as usual, except for the boundary layer effects playing the role usually played by the bed being uneven.

The governing equations (14) for the potential are, in this case

$$
\begin{aligned}
\nabla^{2} \Phi+\frac{1}{\mu^{2}} \frac{\partial^{2} \Phi}{\partial z^{2}} & =0, & -h & \leqslant z \leqslant 0, \\
\frac{1}{\mu^{2}} \frac{\partial \Phi}{\partial z}-\chi \Omega \nabla^{2} \Phi & =0, & z & =-h, \\
-\omega^{2} \Phi+\frac{1}{\mu^{2}} \frac{\partial \Phi}{\partial z} & =0, & z & =0,
\end{aligned}
$$

As usual in WKB, we first consider the potential $\Phi(\mathbf{x}, z)$ written as

$$
\Phi(\mathbf{x}, z)=\mathrm{A}(\mathbf{x}, z) \exp (i \mathrm{~S}(\mathbf{x}, z))
$$

with A and S real functions. Substituting (18) into (17) we get

$$
\begin{array}{rlrl}
\nabla^{2} \mathrm{~A}-\mathrm{A} \boldsymbol{\nabla} \mathrm{S} \cdot \boldsymbol{\nabla} \mathrm{S}+\frac{1}{\mu^{2}}\left(\frac{\partial^{2} \mathrm{~A}}{\partial z^{2}}-\mathrm{A} \frac{\partial \mathrm{S}}{\partial z} \frac{\partial \mathrm{S}}{\partial z}\right) & =0, & & -h \leqslant z \leqslant 0, \\
\frac{\mathrm{A}}{\mu^{2}} \frac{\partial \mathrm{A}}{\partial z}+\chi \Im\{\Omega\} \nabla \cdot\left(\mathrm{A}^{2} \nabla \mathrm{S}\right)- & \\
-\chi \Re\{\Omega\}\left(\mathrm{A} \nabla^{2} \mathrm{~A}-\mathrm{A}^{2} \nabla \mathrm{S} \cdot \nabla \mathrm{S}\right) & =0, & z=-h, \\
-\omega^{2} \mathrm{~A}+\frac{1}{\mu^{2}} \frac{\partial \mathrm{A}}{\partial z} & =0, & z=0,
\end{array}
$$

from the real parts, while from the imaginary parts we get

$$
\begin{array}{rlrl}
\nabla \cdot\left(\mathrm{A}^{2} \nabla \mathrm{S}\right)+\frac{1}{\mu^{2}} \frac{\partial}{\partial z}\left(\mathrm{~A}^{2} \frac{\partial \mathrm{S}}{\partial z}\right) & =0, & & -h \leqslant z \leqslant 0, \\
\frac{\mathrm{A}^{2}}{\mu^{2}} \frac{\partial \mathrm{S}}{\partial z}-\chi \Re\{\Omega\} \nabla \cdot\left(\mathrm{A}^{2} \nabla \mathrm{S}\right)- & & z=-h, \\
-\chi \Im\{\Omega\}\left(\mathrm{A} \nabla^{2} \mathrm{~A}-\mathrm{A}^{2} \nabla \mathrm{S} \cdot \nabla \mathrm{S}\right) & =0, & & z=0 . \\
\frac{\mathrm{A}}{\mu^{2}} \frac{\partial \mathrm{S}}{\partial z} & =0, &
\end{array}
$$


In the above expressions, "S" and " $\Re$ " stand, respectively, for "imaginary part of" and "real part of".

The essence of the WKB approximation is to consider two different horizontal scales, usually one corresponds to the wave and the other to the bottom variations. In this case, the latter will correspond to the boundary layer effects (which are order $\chi$ ). Following usual WKB procedure (see, e.g., Dingemans (1997) for details), we consider the asymptotic expansions

$$
\begin{aligned}
& \mathrm{A}=\alpha_{0}+\chi^{2} \alpha_{1}+\chi^{4} \alpha_{2}+\ldots, \\
& \mathrm{S}=\chi^{-1}\left(\beta_{0}+\chi^{2} \beta_{1}+\chi^{4} \beta_{2}+\ldots\right),
\end{aligned}
$$

The slow variable is now defined as $\hat{\mathbf{x}}=\chi \mathbf{x}$, so that $\boldsymbol{\nabla}=\chi \hat{\boldsymbol{\nabla}}$. We remark that functions $\alpha_{i}$ and $\beta_{i}$ in the above expansions are slowly varying, i.e. $\mathcal{O}\left(\hat{\boldsymbol{\nabla}} \alpha_{i}\right)=$ $\mathcal{O}\left(\hat{\nabla} \beta_{i}\right)=\mathcal{O}(1)$.

Introducing expansions (21) into equations (20), the leading order implies the well known result that $\partial \beta_{0} / \partial z=0$, i.e. $\beta_{0}=\beta_{0}(\mathbf{x})$. Taking this into account, and substituting (21) into equations (19) we get, to the leading order

$$
\begin{array}{rlrl}
-\alpha_{0} \hat{\boldsymbol{\nabla}} \beta_{0} \cdot \hat{\boldsymbol{\nabla}} \beta_{0}+\frac{1}{\mu^{2}} \frac{\partial^{2} \alpha_{0}}{\partial z^{2}} & =0, & -h \leqslant z \leqslant 0, \\
\frac{\alpha_{0}}{\mu^{2}} \frac{\partial \alpha_{0}}{\partial z} & =0, & z & =-h, \\
-\omega^{2} \alpha_{0}+\frac{1}{\mu^{2}} \frac{\partial \alpha_{0}}{\partial z} & =0, & z & =0,
\end{array}
$$

which, at it is well known, implies

$$
\alpha_{0}(\mathbf{x}, z)=a_{\Phi}(\mathbf{x}) f(\mathbf{x}, z), \quad f(\mathbf{x}, z) \equiv \frac{\cosh (\mu k(z+h))}{\cosh (\mu k h)}
$$

where $k$ satisfies the Eikonal equation $k^{2}=\hat{\boldsymbol{\nabla}} \beta_{0} \cdot \hat{\nabla} \beta_{0}$. Further, the expression (22c) implies

$$
\omega^{2}=\frac{k}{\mu} \tanh (\mu k h)
$$

which is the dispersion relationship (in dimensionless form) allowing to compute $k$ as a function of $\omega$ and $h$.

In order to know the spatial evolution of $\alpha_{0}(\mathbf{x}, z)$, i.e., $a_{\Phi}(\mathbf{x})$, the terms order $\mathcal{O}\left(\chi^{2}\right)$ are to be analyzed. Introducing expansions (21) into equation (20) we 
get, to the following order

$$
\begin{aligned}
\hat{\boldsymbol{\nabla}} \cdot\left(\alpha_{0}^{2} \hat{\boldsymbol{\nabla}} \beta_{0}\right)+\frac{1}{\mu^{2}} \frac{\partial}{\partial z}\left(\alpha_{0}^{2} \frac{\partial \beta_{1}}{\partial z}\right) & =0, & & -h \leqslant z \leqslant 0, \\
\frac{\alpha_{0}^{2}}{\mu^{2}} \frac{\partial \beta_{1}}{\partial z}+\Im\{\Omega\} \alpha_{0}^{2} \hat{\boldsymbol{\nabla}} \beta_{0} \cdot \hat{\boldsymbol{\nabla}} \beta_{0} & =0, & z & =-h, \\
\alpha_{0} \frac{\partial \beta_{1}}{\partial z} & =0, & z & =0 .
\end{aligned}
$$

Depth integrating the continuity equation (25a), using the boundary conditions at $z=-h$ and $z=0$, and recalling expression (23), we get

$$
\boldsymbol{\nabla} \cdot\left(a_{\Phi}^{2} \hat{\boldsymbol{\nabla}} \beta_{0} \int_{-h}^{0} f^{2} d z\right)=-\chi \Im\{\Omega\} \frac{a_{\Phi}^{2} k^{2}}{\cosh ^{2}(\mu k h)},
$$

where the boundary layer effects, order $\chi$, appear in the right hand side.

It is well known (see, e.g., Dingemans (1997)) that $\int_{-h}^{0} f^{2} d z=c c_{g}$, where $c$ and $c_{g}$ are the wave and group celerities respectively, given by

$$
c \equiv \frac{\omega}{k}, \quad \text { and } \quad c_{g}=\frac{\partial \omega}{\partial k}=\frac{c}{2}(1+\mathrm{G}),
$$

with

$$
\mathrm{G} \equiv \frac{2 \mu k h}{\sinh (2 \mu k h)}=\frac{2 k^{\prime} h^{\prime}}{\sinh \left(2 k^{\prime} h^{\prime}\right)}
$$

The analysis for the mild slope case is similar. However, the bottom boundary condition introduces an extra term accounting for the bed slope (see expression (14b)). The leading order yields the same results in equations (23) and (24), and now the expression (25b) becomes

$$
\alpha_{0}^{2} \hat{\boldsymbol{\nabla}} \beta_{0} \cdot \hat{\nabla} h+\frac{\alpha_{0}^{2}}{\mu^{2}} \frac{\partial \beta_{1}}{\partial z}+\Im\{\Omega\} \alpha_{0}^{2} \hat{\boldsymbol{\nabla}} \beta_{0} \cdot \hat{\nabla} \beta_{0}=0, \quad z=-h,
$$

but, depth integrating, the expression (26) remains valid.

\section{Discussion of the boundary layer effects}

Summarizing the above results, the WKB approximation yield

$$
\Phi=a_{\Phi} \frac{\cosh (\mu k(z+h))}{\cosh (\mu k h)} \exp \left(i \chi^{-1} \beta_{0}\right)
$$


where $k(\mathbf{x})$ is given by the dispersion expression $(24), \beta_{0}(\mathbf{x})$ satisfies

$$
\hat{\boldsymbol{\nabla}} \beta_{0} \cdot \hat{\nabla} \beta_{0}=k^{2} \quad \text { i.e. } \quad \boldsymbol{\nabla} \beta_{0} \cdot \boldsymbol{\nabla} \beta_{0}=\chi^{2} k^{2},
$$

and the amplitude $a_{\Phi}(\mathbf{x})$ satisfies the equation (26). Besides, recalling expression (16), the free surface satisfies

$$
\eta=i a_{\eta} \exp \left(i\left(\chi^{-1} \beta_{0}\right)\right)
$$

with $a_{\eta} \equiv \omega a_{\Phi}$.

In order to show the influence of the boundary layer on the wave propagation, let us focuss on the behavior of $a_{\eta}$ in the one dimensional case. According to the expression (26), and recalling equation (15),

$$
\boldsymbol{\nabla} \cdot\left(a_{\eta}^{2} c c_{g} \hat{\boldsymbol{\nabla}} \beta_{0}\right)=-\chi \frac{a_{\eta}^{2} k^{2}}{\cosh ^{2}(\mu k h)} \Im\left\{\frac{1+i}{\sqrt{2 \omega}} \vartheta\right\},
$$

so that in the one dimensional case, being $\hat{\nabla} \beta_{0}=\partial \beta_{0} / \partial \hat{x}=k$ and $c k=\omega$,

$$
\frac{\partial}{\partial x}\left(a_{\eta}^{2} c_{g}\right)=-\chi \frac{a_{\eta}^{2} k^{2}}{\omega \cosh ^{2}(\mu k h)} \Im\left\{\frac{1+i}{\sqrt{2 \omega}} \vartheta\right\},
$$

or, alternatively

$$
\frac{\partial}{\partial x}\left(a_{\eta}^{2} c_{g}\right)=-\chi \frac{a_{\eta}^{2} \mathrm{G} \sqrt{\omega}}{h} \Im\left\{\frac{1+i}{\sqrt{2}} \vartheta\right\},
$$

with $\mathrm{G}$ defined in equation (27). In dimensional form, expression (29) reads

$$
\frac{\partial}{\partial x^{\prime}}\left(a_{\eta}^{\prime 2} c_{g}^{\prime}\right)=-\sqrt{\nu_{0}^{\prime} \omega^{\prime}} \frac{a_{\eta}^{\prime 2} \mathrm{G}}{h^{\prime}} \Im\left\{\frac{1+i}{\sqrt{2}} \vartheta\right\}
$$

where $\nu_{0}^{\prime}$ is the already mentioned characteristic kinematic eddy viscosity (or the viscosity if it is considered constant). We remark that the RHS stands for the damping. For the laminar case, $\vartheta=1$, the above expression is equivalent to the results presented in Dalrymple et al. (1984). According to the definition of $\mathrm{G}$, this term rapidly decreases in deep water $\left(k^{\prime} h^{\prime} \gg 1\right)$. If viscous effects are ignored, the well know energy conservation expression $a_{\eta}^{\prime 2} c_{g}^{\prime}=c t t$ is recovered.

Further, potential and free surface Fourier components read, in dimensional form and expressed using the wave amplitude $a_{\eta}^{\prime}$,

$$
\Phi^{\prime}=\frac{g^{\prime}}{\omega^{\prime}} a_{\eta}^{\prime} f \exp \left(i\left(\int k^{\prime} d x^{\prime}+\delta\right)\right)
$$

and

$$
\eta^{\prime}=i a_{\eta}^{\prime} \exp \left(i\left(\int k^{\prime} d x^{\prime}+\delta\right)\right)
$$


¿From the potential, the horizontal velocity is

$$
u^{\prime} \equiv \frac{\partial \Phi^{\prime}}{\partial x^{\prime}}=\frac{g^{\prime}}{\omega^{\prime}}\left(\frac{\partial\left(a_{\eta}^{\prime} f\right)}{\partial x^{\prime}}+i k^{\prime} a_{\eta}^{\prime} f\right) \exp \left(i\left(\int k^{\prime} d x^{\prime}+\delta\right)\right)
$$

Comparing expressions (32) and (31b), velocity and free surface elevation are slightly out of phase due to the term

$$
\frac{\partial\left(a_{\eta}^{\prime} f\right)}{\partial x^{\prime}}
$$

that stands for both, depth variations and bottom boundary layer effects.

To illustrate some of the above results, we shall focus on the constant viscosity case (i.e., laminar or constant eddy viscosity). For constant eddy viscosity, $\nu_{0}^{\prime}$ is a constant given value and, according to expression (11), $\vartheta=1$.

We consider a monochromatic wave train with a period of 8 seconds propagating over a 500 meters distance (Figure 3), the depth varying linearly from 10 to 2 meters. Figure 3 shows also the evolution of $c_{g}^{\prime}$, since it is important in shoaling aspects according to expression (30). Note that, because $c_{g}^{\prime}$ decreases in the propagation direction, shoaling is expected.

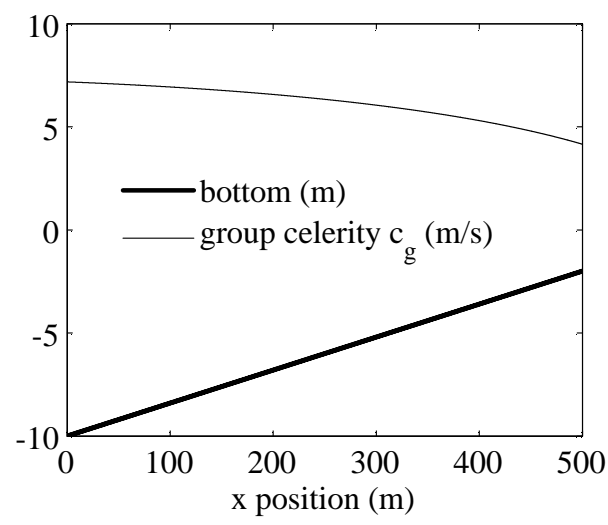

Fig. 3. Evolution of the group celerity across 500 meters propagation. The incident wave is composed only by one component with a period of 8 seconds.

Figure 4 compares the results for the wave amplitude obtained with and without boundary layer. A constant eddy viscosity $\nu_{0}^{\prime}=10^{-4} \mathrm{~m}^{2} / \mathrm{s}$ has been used. As depicted from the figure, boundary layer reduces the wave amplitude as long as waves propagates: in this case, however, shoaling due to depth variations are stronger than boundary layer effects. 


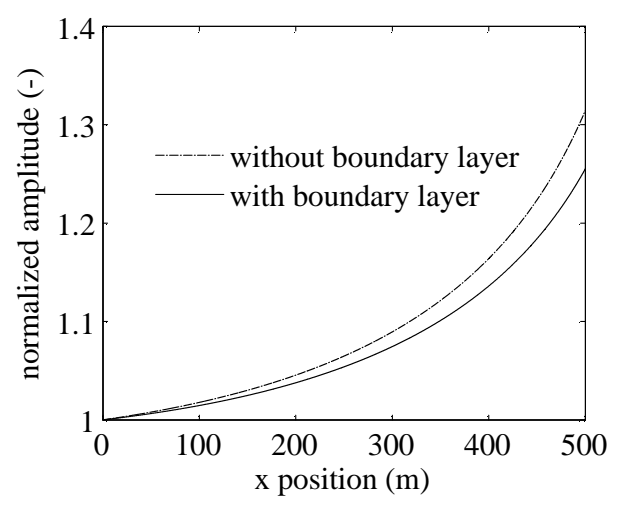

Fig. 4. Normalized wave amplitude along the distance considering the damping term in Equation (30) (solid line) and neglecting the viscous effects (dashed line).

\section{Concluding remarks}

A formulation for linear wave propagation with boundary layer effects has been presented. The boundary layer effects are introduced in the wave propagation boundary value problem through a modification of the bottom boundary layer. The model can be applied with either laminar or turbulent eddy viscosity. The boundary layer effects appear as a modification of the energy equation where a new term accounts for the effects of the bottom friction. The results have been here presented in a one dimensional case, but it can be easily implemented to 2 dimensional linear wave propagation problems in an efficient computational way.

\section{Acknowledgments}

The authors would like to thank financial support from MEC thought project CTM2006-12072. 


\section{References}

Booij, N., 1981. Gravity Waves on Water with Non-Uniform Depth and Current. PhD. Thesis, Technical University of Delft, The Nederlands.

Dalrymple, R. A., Kirby, J. T., Hwang, P. A., 1984. Wave diffraction due to areas of energy dissipation. Journal of Waterway, Por, Coastal and Ocean Engineering 110, 67-79.

Dingemans, M. W., 1997. Water wave propagation over uneven bottoms. World Scientific, Singapore.

Fredsoe, J., Deigaard, R., 1992. Mechanics of coastal sediment transport. World Scientific, New York, New York.

Grant, W. D., Madsen, O. S., 1979. Combined wave and current interaction with a rough bottom. Journal of Geophysical Research 84(C4), 1797-1808.

Kajiura, K., 1964. On the bottom friction in an oscillatory current. Bull. Eqrthquake Res. Int. 42, 147-174.

Liu, P. L.-F., Orfila, A., 2004. Viscous effects on transient long wave propagation. Journal of Fluid Mechanics 520, 83-92.

Liu, P. L.-F., Simarro, G., Vandever, J., Orfila, A., 2006. Experimental and numerical investigations of viscous effects on solitary wave propagation in a wave tank. Coastal Engineering 520(2-3), 181-190.

Nielsen, P., 1992. Coastal bottom boundary layers and sediment transport. World Scientific, Singapore.

Orfila, A., Simarro, G., Liu, P. L.-F., 2007. Bottom frictional effects on periodic long wave propagation. Coastal Engineering, 54, 11, 856-864.

Simarro, G., Orfila, A., 2007. Boundary layer effects on the propagation of weakly nonlinear long waves. In Nonlinear wave dynamics. World Scientific.

Simarro, G., Orfila, A., Liu, P. L.-F., 2008. Bed shear stress under wavecurrent turbulent boundary layer. Journal of Hydraulic Engineering, 134, 2, 225-230.

Svendsen, I. A., 2005. Introduction to nearshore hydrodynamics. Advanced Series on Ocean Engineering. World Scientific, Singapore. 